\title{
On the Weak Measurement of Velocity in Bohmian Mechanics*
}

\author{
Detlef Dürr $†$ Sheldon Goldstein \\ and Nino Zanghì ${ }^{\S}$
}

August 13, 2008

\begin{abstract}
In a recent article [1], Wiseman has proposed the use of so-called weak measurements for the determination of the velocity of a quantum particle at a given position, and has shown that according to quantum mechanics the result of such a procedure is the Bohmian velocity of the particle. Although Bohmian mechanics is empirically equivalent to variants based on velocity formulas different from the Bohmian one, and although it has been proven that the velocity in Bohmian mechanics is not measurable, we argue here for the somewhat paradoxical conclusion that Wiseman's weak measurement procedure indeed constitutes a genuine measurement of velocity in Bohmian mechanics. We reconcile the apparent contradictions and elaborate on some of the different senses of measurement at play here.
\end{abstract}

\section{Introduction}

According to the uncertainty principle, it is impossible to simultaneously measure both the position and the velocity of a quantum particle, at least not to arbitrary

${ }^{*}$ This paper is dedicated to Jürg Fröhlich on the occasion of one of his birthdays.

${ }^{\dagger}$ Mathematisches Institut, Ludwig-Maximilians-Universität, Theresienstr. 39, 80333 München, Germany. Email: duerr@mathematik.uni-muenchen.de

${ }^{\ddagger}$ Departments of Mathematics, Physics and Philosophy, Hill Center, Rutgers, The State University of New Jersey, 110 Frelinghuysen Road, Piscataway, NJ 08854-8019, USA. E-mail: oldstein@math.rutgers.edu

${ }^{\S}$ Dipartimento di Fisica dell'Università di Genova and INFN sezione di Genova, Via Dodecaneso 33, 16146 Genova, Italy. E-mail: zanghi@ge.infn.it 
accuracy. The basic reason for this limitation is that in quantum mechanics a measurement of the position of a particle to a given accuracy produces a corresponding narrowing of its wave function and hence a corresponding increase in the uncertainty about its velocity. Moreover, this is as true of Bohmian mechanics, a version of quantum mechanics in which a particle always has a velocity as well as a position, as it is of orthodox quantum theory, in which it does not. This suggests that in order to measure the velocity of a Bohmian particle at a given position, it might be good to exploit a measurement procedure that somehow does not significantly affect the wave function of the particle.

Such a procedure, a so-called weak measurement, has been developed by Aharonov, Albert, and Vaidman [2]. And Howard Wiseman has indeed proposed in a recent article[1] that weak measurements be used to measure the velocity of a particle at a given position.

More precisely, Wiseman invokes the theory of weak measurements to provide an "operational definition for the velocity for a particle at position $x$ ":

$$
v(x) \equiv \lim _{\tau \rightarrow 0} \tau^{-1} \mathrm{E}\left[x_{\text {strong }}(\tau)-x_{\text {weak }} \mid x_{\text {strong }}(\tau)=x\right] .
$$

He then observes that this quantity is precisely the velocity that defines Bohmian mechanics $[3,4,5,6]$, and uses this fact to respond to some objections that have been raised against it.

In this formula $x_{\text {weak }}$ and $x_{\text {strong }}$ denote respectively the results of a weak measurement of the position of the particle at some time, say $t=0$, and a strong measurement of the position a short time $\tau$ later. The expectation symbol $\mathrm{E}$ in the formula refers to the average over a large ensemble of systems, all prepared in the same initial state $\psi$ at time 0 , and for all of which the result of a strong measurement of position at time $\tau$, following the weak measurement at time 0 , is $x$.

A strong measurement of an observable $\hat{A}$ is just a standard quantum measurement of the observable - one which collapses the wave function of the system involved to the eigenstate of $\hat{A}$ corresponding to the eigenvalue found in the measurement. The average of such values for a large ensemble of systems in the state $\psi$ is, of course, $\langle\psi|\hat{A}| \psi\rangle$. In contrast, a weak measurement of $\hat{A}$ (which will be described in more detail in the next section) does not collapse the wave function of the system, and in fact is such that the change in the wave function that is produced by the procedure can be made arbitrarily small. The price to be paid for this desirable feature is that very little information about the system is obtained in a single such measurement, the result found reflecting mainly the effect of noise introduced by the procedure rather than any property of the system itself. Nonetheless, the ensemble average for such a procedure is $\langle\psi|\hat{A}| \psi\rangle$, just as for a strong measurement. 
Weak measurements are most interesting when combined with post-selection: Consider the subensemble for which, after the weak measurement, the system is found in state $\varphi$ at time $\tau>0$. When $\tau=0+$, the average over this subensemble of the result of the weak measurement at time 0 is of course still $\langle\psi|\hat{A}| \psi\rangle$ when $\varphi=\psi$, and in general is given by the so-called weak value $[2]^{1}$

$$
\left\langle\hat{A}_{\mathrm{w}}\right\rangle_{|\psi\rangle}=\operatorname{Re} \frac{\langle\varphi|\hat{A}| \psi\rangle}{\langle\varphi \mid \psi\rangle} .
$$

For $\tau>0$ the value of the subensemble average of course involves the unitary evolution operator $U(\tau)$ for time $\tau$ and is given by the weak value

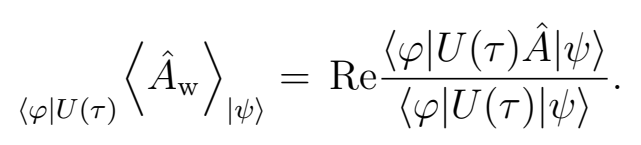

In terms of this, the velocity definition (1) becomes

$$
v(x)=\lim _{\tau \rightarrow 0} \tau^{-1}\left[x-\operatorname{Re} \frac{\langle x|U(\tau) \hat{X}| \psi\rangle}{\langle x|U(\tau)| \psi\rangle}\right],
$$

where $\hat{X}$ is the position operator of the particle. One easily computes with $U(\tau)=$ $\exp (-i \hat{H} \tau / \hbar)$ and $\hat{H}=\hat{p}^{2} / 2 m+V(\hat{x})$ that (4) becomes

$$
v(x)=v^{\psi}(x) \equiv \frac{j^{\psi}(x)}{|\psi(x)|^{2}}
$$

with

$$
j^{\psi}(x)=(\hbar / m) \operatorname{Im} \psi(x) \nabla \psi(x),
$$

the usual quantum flux. (5) is the expression for the Bohmian velocity:

$$
\dot{X}(t)=v^{\psi}(X(t), t)=\frac{j^{\psi}(X(t) ; t)}{|\psi(t, X(t))|^{2}} .
$$

This equation, together with Schrödinger's equation for the wave function, is the defining dynamical equation of Bohmian mechanics for a single particle, with a similar equation for the Bohmian mechanics of a many-particle system.

Wiseman does not claim, either in the above quotation or anywhere else in his article, that his weak measurement procedure, providing an "operational definition

\footnotetext{
${ }^{1}$ Our usage here is that of Wiseman [1]. It is a bit different from that of [2], which refers to the ratio following "Re" in equation (2), which could be complex, as the weak value.
} 
for the velocity," actually measures the Bohmian velocity. There is a good reason for this: There are variants of Bohmian mechanics based on velocity formulas different from $(5,6)$ that yield theories empirically equivalent to Bohmian mechanics. The existence of a procedure to measure the velocity would seem to contradict this empirical equivalence.

We elaborate. Recall the quantum continuity equation

$$
\partial_{t}|\psi(x, t)|^{2}=-\operatorname{div} j^{\psi}(x, t) \text {. }
$$

From this equation $j^{\psi}$ is not uniquely defined: A divergence free vector can be added without affecting the continuity equation. In [7] some "physically reasonable" additions are discussed, giving rise to (empirically equivalent) variants of Bohmian mechanics which have different velocity fields (7) with $j^{\psi}$ replaced by the new $j^{\prime} \psi$. With respect to this Wiseman [1] describes his findings as follows:

"...a particular $\mathbf{j}$ is singled out if one requires that $\mathbf{j}$ be determined $e x$ perimentally as a weak value, using a technique that would make sense to a physicist with no knowledge of quantum mechanics. This "naively observable" $\mathbf{j}$ seems the most natural way to define $\mathbf{j}$ operationally. Moreover, I show that this operationally defined $\mathbf{j}$ equals the standard $\mathbf{j}$, so, assuming $\dot{\mathbf{x}}=\mathbf{j} / P$ one obtains the dynamics of BM. It follows that the possible Bohmian paths are naively observable from a large enough ensemble."

Notice that Wiseman claims only that the Bohmian paths (or Bohmian velocities) are "naively observable," but not that they are genuinely observable. He claims not that the current (and the velocity associated with it) found in his procedure is ipso facto the actual current, but only that it is "the most natural way to define $\mathbf{j}$ operationally." In short, Wiseman does not claim that his procedure, which we shall call a "weak measurment of velocity," provides a genuine measurement of velocity.

But, as we shall argue, it does - despite the apparent contradiction. In more detail, we shall be concerned in this paper with the following statements:

(1) A "weak measurement of velocity" in Bohmian mechanics is, in a reasonable sense, a genuine measurement of velocity.

(2) The same thing is true for the variants of Bohmian mechanics based on a velocity formula different from the Bohmian one mentioned above. 
(3) Bohmian mechanics and the variants referred to in (2) are empirically equivalent to each other - and to standard quantum mechanics. In particular, for all of them the result of a "weak measurement of velocity" is given by the Aharonov-Albert-Vaidman formula given above, and hence by the formula for velocity in Bohmian mechanics.

(4) It is impossible to measure the velocity in Bohmian mechanics [6].

There is of course an obvious contradiction between the first three of these statements. A genuine measurement of velocity must reveal the velocity and hence could be used to empirically distinguish the theories based on different velocity formulas. And if a theory is based on a velocity formula different from the Bohmian one, a genuine velocity measurement for the theory can't yield the Bohmian velocity.

At least one of these three statements must be false. However (3) is well established, and true. (The reason for this is basically that in Bohmian mechanics and its variants the statistics for the results of experiments - including weak measurements are determined by the same $|\Psi|^{2}$ probabilities as for orthodox quantum theory.) In Section 2 we shall show that (1) is also correct. We shall do this by an analysis that seems to apply to the variants of Bohmian mechanics referred to in (2), as well as to Bohmian mechanics itself. Thus in Section 2 it shall seem as if we establish (2) as well as (1). In Section 3 we shall explain why the analysis in Section 2 is in fact incorrect for the alternatives to Bohmian mechanics, so that (2) is not established by the analysis yielding (1) - a good thing since (2) is false.

We shall also examine, in Section 4, the crucial condition responsible for the success of Bohmian mechanics here, showing directly that this condition indeed uniquely characterizes Bohmian mechanics. Finally, in Section 5, we address the apparent contradiction between statements (1) and (4).

\section{Bohmian Analysis of Weak Measurement of Velocity}

We now consider a Bohmian particle with wave function $\psi$ at time 0 . We model the measurement apparatus which measures weakly the position of the Bohmian particle by a pointer and we denote the actual Bohmian pointer position by $Y$. We denote by $X(t)$ the position of the Bohmian particle. We measure $X=X(0)$ weakly at time 0 and very shortly after that, at time $\tau$, we perform a strong measurement of $X(\tau)$.

Let us spell out what this means. Let $\Phi=\Phi(y)$ be the wave function of the apparatus in its ready state, with the pointer-variable $Y$ centered at $Y=0$ : $\Phi$ 
is a real wave packet of spread $\sigma$-i.e., such that $\Phi(y)=\phi(y / \sigma)$ with $\phi$ fixed as $\sigma \rightarrow \infty$-for which the expected value of $Y$ vanishes, ${ }^{2}$

$$
\int d y y|\Phi(y)|^{2}=0
$$

for example,

$$
\Phi(y) \sim e^{-\frac{y^{2}}{4 \sigma^{2}}}
$$

The weak measurement begins at time 0 with an interaction between system and apparatus that leads to the following instantaneous transition from initial quantum state to final quantum state:

$$
\psi(x) \Phi(y) \rightarrow \psi(x) \Phi(y-x)
$$

(corresponding in ket notation to

$$
\int d x \psi(x)|x\rangle|\Phi\rangle \rightarrow \int d x \psi(x)|x\rangle|\Phi\rangle_{x}
$$

where |\rangle$_{x}$ indicates translation by $x$ ). Immediately after this, the pointer position is measured and recorded. When the measured value is $Y$, (up to normalization) the system wave function after the measurement is, by the projection postulate,

$$
\psi_{0+}(x)=\psi_{Y}(x) \equiv \psi(x) \Phi(Y-x)
$$

Note that since the result $Y$ is random, with probability distribution

$$
\rho^{Y}(y)=\int d x|\psi(x)|^{2}|\Phi(y-x)|^{2}
$$

the system wave function $\psi_{0+}(x)$ is random as well. (In Bohmian mechanics this wave function is called the conditional wave function [6].)

For a standard von Neumann measurement of position, the spread $\sigma$ of the apparatus wave function $\Phi$ is taken very small so that the wave function (13) is an approximate eigenstate of the position operator concentrated near the value $x=Y$. But in a weak measurement the pointer wave function $\Phi(y)$ is very spread out $(\sigma$

\footnotetext{
${ }^{2}$ This condition on the initial apparatus state $\Phi$, assumed for our analysis of the weak measurement of velocity in Bohmian mechanics, is weaker than what is in general needed for the result of a weak measurement, with post-selection and averaging, to be given by (2), namely that $\phi$ be real and even, or real and odd.
} 
is very large), varying on the scale $\sigma$, whereas $\psi(x)$ varies on a scale of order unity (near say 0 ). We thus should have from (13) that

$$
\psi_{Y}(x) \approx \Phi(Y) \psi(x)
$$

and that up to normalization

$$
\psi_{0+}(x) \approx \psi(x)
$$

with small error, of order $1 / \sigma$. Although a single weak measurement does not measure the actual position of the particle, by averaging over a large sample of identical experiments one obtains information about the mean value of position; we have, observing (10)

$$
\mathbb{E}(Y) \equiv \int y \rho^{Y}(y) d y=\int x \rho^{X}(x) d x \equiv \mathbb{E}(X)
$$

where $\rho^{X}(x)=|\psi(x)|^{2}$.

The conditional probability density of $Y$ given $X=x$ is

$$
\rho^{Y}(y \mid X=x)=\frac{\rho^{X, Y}(x, y)}{\rho^{X}(x)}=\frac{|\psi(x)|^{2}|\Phi(y-x)|^{2}}{|\psi(x)|^{2}}=|\Phi(y-x)|^{2},
$$

and hence in a weak measurement

$$
\mathbb{E}(Y \mid X=x) \equiv \int y \rho^{Y}(y \mid X=x)=x
$$

The "weak measurement of velocity" is completed by performing at time $\tau$, on each member of the ensemble, a (strong) measurement of the position of the particle, and taking the conditional average indicated by (1). Conditioning on the event that $X(\tau)=x$, the Bohmian version of this conditional average is

$$
\lim _{\tau \rightarrow 0} \frac{1}{\tau} \mathbb{E}(x-Y \mid X(\tau)=x)=\lim _{\tau \rightarrow 0} \frac{1}{\tau}(x-\mathbb{E}(Y \mid X(\tau)=x)) .
$$

To see how the Bohmian velocity comes in we note, writing $X$ for $X(0)$, that

$$
X(\tau) \approx X+v^{\psi_{0+}} \tau
$$

When $\tau \rightarrow 0$ the error of this approximation is of smaller order than $\tau$. By (16) we have that

$$
v^{\psi_{0+}} \approx v^{\psi}(x)
$$

and hence that

$$
X(\tau) \approx X+v^{\psi}(X) \tau \approx X+v^{\psi}(X(\tau)) \tau
$$


With this approximation we can identify the event $X(\tau)=x$ with the event $X=$ $x-v^{\psi}(x) \tau$. Therefore by (18)

$$
\mathbb{E}(Y \mid X(\tau)=x) \approx \mathbb{E}\left(Y \mid X=x-v^{\psi}(x) \tau\right)=x-v^{\psi}(x) \tau
$$

and thus for (19) we obtain

$$
\lim _{\tau \rightarrow 0} \frac{1}{\tau}(x-\mathbb{E}(Y \mid X(\tau)=x)) \approx v^{\psi}(x),
$$

with the approximation becoming exact for a weak measurement, i.e., in the limit $\sigma \rightarrow \infty$.

The details of this analysis, in particular equations (18), (22) and (24), show that in a "weak measurement of velocity" in Bohmian mechanics the result of the averaging is $v^{\psi}$ precisely because the Bohmian particle had velocity $v^{\psi}$. We are thus justified in asserting that for Bohmian mechanics this procedure of weak measurement genuinely measures the Bohmian velocity. Now we ask: What is specifically "Bohmian" in formulas (18), (22) and (24)? The answer, it would seem, is nothing. These formulas seem to hold for all variants of Bohmian mechanics which have differentiable paths. But that is incompatible with the (correct) weak measurement formula (4) that yields the Bohmian velocity. But where is the mistake? The answer is in fact not easy to find and lies in scrutinizing more carefully weak measurements.

\section{A More Careful Analysis}

Now here is the catch: Since (16) is only approximately satisfied for $\sigma$ large, where $\sigma$ is the spread of $\Phi(y)$, we cannot in general dismiss the possibility that $v$ depends also on $Y$. The $Y$ dependence of the conditional wave function $\psi_{0+}(x)=\psi(x) \Phi(Y-x)$ yields in general a $Y$ dependence of the induced velocity field. ${ }^{3}$ Therefore after the weak measurement we have truthfully now $v^{\psi_{0+}}=v(x, Y)$ and thus for (20) we have

$$
X(\tau) \approx X+v(X(\tau), Y) \tau .
$$

\footnotetext{
${ }^{3}$ We note that the $Y$ dependence of the velocity of the particle can be simply computed from the velocity formula which the Bohmian type theory provides given the wave function of the entire system consisting of particle and apparatus. There is thus no need to introduce the conditional wave function. The conditional wave function focuses however on the source of the $Y$-dependence: The weak measurement does affect the wave function-if only a tiny bit. That tiny bit changes the velocity a tiny bit, having possibly a big effect in weak measurements.
} 
as a better approximation than (22), exact to order $\tau$, and the left hand sides of $(24)$ becomes

$$
\lim _{\tau \rightarrow 0} \frac{1}{\tau}(x-\mathbb{E}(Y \mid X=x-v(x, Y) \tau)) .
$$

This expression is not anymore easy to handle and in general it is not equal to $v^{\psi}$. Before entering into more details we make the trivial observation that if the weak measurement were such that $v(x, Y)=v^{\psi}(x)$, i.e., if

$$
v^{\psi \Phi_{y}}=v^{\psi}
$$

where $\Phi_{y}(x)=\Phi(y-x)$, the analysis of Section 2 would be correct and the "weak measurement of velocity" would indeed be a genuine measurement of velocity yielding the result $v^{\psi}$. When (27) is satisfied, as is the case for Bohmian mechanics, we have in fact by (17) that, to order $\tau$,

$$
\rho^{Y}(y \mid X(\tau)=x)=\rho^{Y}\left(y \mid X=x-v^{\psi}(x) \tau\right)=|\Phi|^{2}\left(y-\left[x-v^{\psi}(x) \tau\right]\right) .
$$

With a variant of Bohmian mechanics, however, the velocity need not (in fact, will not, see below) obey (27) exactly . Rather (27) will hold only approximately, presumably with an error of order $1 / \sigma$ since $\Phi$ varies on scale $\sigma$. At first sight one might be inclined to ignore this error. However one must be careful here, since in a weak measurement a large quantity (here $Y$, of order $\sigma$ ) is averaged to yield (because of near-perfect cancellation) a result of order unity. And a small change in the probability distribution involved could lead to an effect of order unity as well.

Indeed, an order $1 / \sigma$ error in (27) would be expected to yield an additional contribution to (28) of order $\tau / \sigma$, yielding a contribution of order $\tau$ to $\mathbb{E}(Y \mid X(\tau)=x)$ (since $Y$ is of order $\sigma$ ), and hence a contribution of order unity in (19). These expectations are correct.

Indeed, writing now $v_{B}^{\psi}$ for the velocity (5) in Bohmian mechanics, we have that (to order $\tau$ )

$$
\begin{aligned}
\rho^{Y}(y \mid X(\tau)=x) & =|\Phi|^{2}\left(y-\left[x-v_{B}^{\psi}(x) \tau\right]\right) \\
& \left.=|\Phi|^{2}\left(y-\left[x-v^{\psi}(x) \tau\right]-\left[v^{\psi}(x)-v_{B}^{\psi}(x)\right] \tau\right]\right) \\
& \approx|\Phi|^{2}\left(y-\left[x-v^{\psi}(x) \tau\right]\right)+\left(v^{\psi}(x)-v_{B}^{\psi}(x)\right) \tau \cdot \nabla_{x}|\Phi|^{2}(y-x)
\end{aligned}
$$

and since $\Phi(y)$ varies on scale $\sigma$ the second term on the last line is of order $\tau / \sigma$. Thus whenever the velocity $v^{\psi}$ is non-Bohmian, and in particular whenever (27) is not obeyed, the conditional distributution of $Y$ given $X(\tau)$ is sufficiently affected so as to vitiate the analysis of Section 2. When the velocity is non-Bohmian the "weak measurement of velocity" fails because of these errors to be a genuine measurement of velocity. 


\section{Bohmian Mechanics and the Crucial Condition}

There is one issue that might still be puzzling here. We have seen that a "weak measurement of velocity" is in fact a genuine measurement of velocity whenever the condition (27) is satisfied. And in this case the velocity found must be the Bohmian velocity. This implies that (27) can be satisfied only for Bohmian mechanics - that it characterizes Bohmian mechanics among all of its variants. We shall now provide a more general and precise formulation of this conclusion, as well as a direct argument for it:

Suppose $v^{\psi}$ defines a variant of Bohmian mechanics for which the condition

$$
v^{\psi \phi}=v^{\psi}
$$

holds for all (differentiable) real-valued functions $\phi$, or at least for a collection of such functions that is "gradient-total," i.e., such that at every point $x \in \mathbb{R}^{3}$, the collection of vectors $\nabla \phi(x)$ spans $\mathbb{R}^{3}$. Then $v^{\psi}=v_{B}^{\psi}$

A similar conclusion holds for a more general configuration space than $\mathbb{R}^{3}$, for example for $\mathbb{R}^{3 N}$. We note that for any fixed (differentiable) real-valued function $\Phi$ that vanishes at $\infty$ (but is not identically 0 ), the collection of functions $\Phi_{y}(x)=$ $\Phi(y-x), y \in \mathbb{R}^{3}$, is gradient-total, since otherwise there would be a direction in which $\Phi$ does not vary. [Note also that for particles without spin, i.e., when $\psi$ is complex-scalar-valued, then the condition (30) amounts basically to requiring that $v^{\psi}$ depend only on the phase $S$ of $\psi$ (arising from the polar decomposition $\psi=R e^{i S / \hbar}$ ).]

Here is the proof: Recall that any variant of Bohmian mechanics, with velocity $v^{\psi}$, must obey the continuity equation, see (8),

$$
\partial_{t}|\psi(x, t)|^{2}=-\operatorname{div}\left(v^{\psi}(x, t)|\psi(x, t)|^{2}\right) .
$$

Consider two such velocity functionals, $v_{1}^{\psi}$ and $v_{2}^{\psi}$. Since they both are such that (31) is obeyed, we have that

$$
\operatorname{div}\left(j_{1}^{\psi}-j_{2}^{\psi}\right)=0
$$

where $j_{i}^{\psi}=|\psi|^{2} v_{i}^{\psi}$. If they both also obey (30), we have that

$$
j_{i}^{\psi \phi}=|\phi|^{2} j_{i}^{\psi}
$$

Then from (32), with $\psi$ replaced by $\psi^{\prime}=\psi \phi$, we have that

$$
\operatorname{div}\left[|\phi|^{2}\left(j_{1}^{\psi}-j_{2}^{\psi}\right)\right]=0
$$


and since

$$
\operatorname{div}\left[|\phi|^{2}\left(j_{1}^{\psi}-j_{2}^{\psi}\right)\right]=|\phi|^{2} \operatorname{div}\left(j_{1}^{\psi}-j_{2}^{\psi}\right)+\nabla|\phi|^{2} \cdot\left(j_{1}^{\psi}-j_{2}^{\psi}\right)=\nabla|\phi|^{2} \cdot\left(j_{1}^{\psi}-j_{2}^{\psi}\right)
$$

it follows that

$$
\nabla|\phi|^{2} \cdot\left(j_{1}^{\psi}-j_{2}^{\psi}\right)=0
$$

Thus if the relevant collection of functions $\phi$ is gradient-total, we have that

$$
j_{1}^{\psi}=j_{2}^{\psi}
$$

and hence that

$$
v_{1}^{\psi}=v_{2}^{\psi}
$$

Since $v_{B}^{\psi}$ is a possible choice for $v^{\psi}$ the conclusion follows.

\section{The Impossibility of Measuring the Velocity in Bohmian Mechanics}

We have argued that by using weak measurements it is possible to measure the velocity of a particle in Bohmian mechanics. We will now reconcile this with the proven impossibility of measuring the velocity in Bohmian mechanics [6], in the sense that no measurement procedure involving an interaction between a particle and any sort of apparatus can yield a result that conveys (with arbitrary precision) the velocity of the particle just prior to the beginning of the procedure.

Let us first note that our weak measurement of velocity can, it seems, be regarded as just such a procedure. It involves an ensemble of systems, each with the same wave function. We can regard one member of the ensemble as the special particle whose velocity is to be measured, with the other members of the ensemble constituting (part of) the apparatus. The selection of subensemble and averaging corresponding to (24), with $x$ the position of the special particle at time $\tau$, then conveys the velocity of the special particle to arbitrary accuracy.

This procedure, however, is not of the sort contemplated in [6]. It is assumed there that neither the initial state $\Psi_{\text {app }}$ of the apparatus nor the interaction $H_{\text {int }}$ between system and apparatus depends on the initial state $\psi$ of the system. (This is reasonable since the whole point of a measurement is to obtain information about a system that would not otherwise be available.) Such measurements have been called linear measurements, in contrast with the nonlinear measurements in which either the initial state of the apparatus $\Psi_{\text {app }}=\Psi^{\psi}$ or the interaction $H_{\text {int }}=H^{\psi}$ depends upon the state $\psi$ of the system. 
The weak measurement of velocity discussed in this paper is clearly nonlinear, ${ }^{4}$ and is thus not precluded by the impossibility claim of [6]. It should nonetheless be contrasted with another nonlinear measurement of velocity for Bohmian mechanics: perform a standard position measurement and plug the result into the formula (5) to obtain the corresponding velocity. While it is difficult to take the latter "measurement of velocity" seriously, and to regard it as anything more than cheating, the weak measurement of velocity in Bohmian mechanics is, as we have argued, a genuine measurement of velocity, even though it is nonlinear.

\section{Conclusion}

Measurement is a tricky and complicated business. Even when, as with Bohmian mechanics and its variants, there is something to be measured, one must be careful. With orthodox quantum theory and the "measurement" of operators as observables, the situation is even more dangerous. We conclude by quoting Bell [4, page 166] on this:

A final moral concerns terminology. Why did such serious people take so seriously axioms which now seem so arbitrary? I suspect that they were misled by the pernicious misuse of the word 'measurement' in contemporary theory. This word very strongly suggests the ascertaining of some preexisting property of some thing, any instrument involved playing a purely passive role. Quantum experiments are just not like that, as we learned especially from Bohr. The results have to be regarded as the joint product of 'system' and 'apparatus,' the complete experimental set-up. But the misuse of the word 'measurement' makes it easy to forget this and then to expect that the 'results of measurements' should obey some simple logic in which the apparatus is not mentioned. The resulting difficulties soon show that any such logic is not ordinary logic. It is my impression that the whole vast subject of 'Quantum Logic' has arisen in this way from the misuse of a word. I am convinced that the word 'measurement' has now been so abused that the field would be significantly advanced by banning its use altogether, in favour for example of the word 'experiment.'

\footnotetext{
${ }^{4}$ Were it possible to clone the wave function $\psi$, the ensemble could have been produced as part of an overall linear measurement, but cloning is not possible [8].
} 
Acknowledgments. The work of S. Goldstein was supported in part by NSF Grant DMS-0504504. That of N. Zanghì was supported in part by INFN.

\section{References}

[1] H. M. Wiseman: Grounding Bohmian mechanics in weak values and Bayesianism, New Journal of Physics 9, (2007) 165, doi:10.1088/1367-2630/9/6/165

[2] Y. Aharonov, D. Z. Albert, and L. Vaidman: How the result of a measurement of a component of the spin of a spin-1/2 particle can turn out to be 100, Phys. Rev. Lett. 60, 1351-1354 (1988)

[3] D. Bohm: A suggested interpretation of the quantum theory in terms of "hidden" variables, Part I, Phys. Rev. 85, 166-179 (1952). D. Bohm: A suggested interpretation of the quantum theory in terms of "hidden" variables, Part II, Phys. Rev. 85, 180-193 (1952)

[4] J.S. Bell: Speakable and unspeakable in quantum mechanics (Cambridge University Press, Cambridge, 1987)

[5] D. Dürr, S. Goldstein, and N. Zanghì: Quantum equilibrium and the origin of absolute uncertainty, J. Statist. Phys. 67, 843-907 (1992), quant-ph/0308039

[6] D. Dürr, S. Goldstein, and N. Zanghì: Quantum equilibrium and the role of operators as observables in quantum theory, J. Statist. Phys. 116, 959-1055 (2004), quant-ph/0308038

[7] E. Deotto and G. C. Ghirardi: Bohmian mechanics revisited, Found. Phys. 28, $1-30$ (1998)

[8] G. C. Ghirardi: Private communication, 1981 